Europhysics Letters

PREPRINT

\title{
Elasticity-driven Nanoscale Texturing in Complex Electronic Materials
}

\author{
A.R. Bishop ${ }^{1}$ T. Lookman ${ }^{1}$, A. Saxena ${ }^{1}$ and S.R. Shenoy ${ }^{2}$ \\ 1 Theoretical Division, Los Alamos National Laboratory, Los Alamos,NM, 87544 USA \\ 2 International Centre for Theoretical Physics, Trieste 34014, Italy
}

PACS. 71.38. $-\mathrm{k}-$.

PACS. 75.47.Gk - .

PACS. 74.72. $-\mathrm{h}-$.

\begin{abstract}
Finescale probes of many complex electronic materials have revealed a nonuniform nanoworld of sign-varying textures in strain, charge and magnetization, forming meandering ribbons, stripe segments or droplets. We introduce and simulate a Ginzburg-Landau model for a structural transition, with strains coupling to charge and magnetization. Charge doping acts as a local stress that deforms surrounding unit cells without generating defects. This seemingly innocuous constraint of elastic 'compatibility', in fact induces crucial anisotropic long-range forces of unit-cell discrete symmetry, that interweave opposite-sign competing strains to produce polaronic elasto-magnetic textures in the composite variables. Simulations with random local doping below the solid-solid transformation temperature reveal rich multiscale texturing from induced elastic fields: nanoscale phase separation, mesoscale intrinsic inhomogeneities, textural cross-coupling to external stress and magnetic field, and temperature-dependent percolation. We describe how this composite textured polaron concept can be valuable for doped manganites, cuprates and other complex electronic materials.
\end{abstract}

High resolution microscopies of many classes of complex electronic materials such as cuprates, manganites, ferroelastic martensites, and relaxor ferroelectric titanates [1, 2, 3, 4 have revealed previously unsuspected, and puzzling, multiscale modulations of charge, spin, polarization and strain variables in stripe- or droplet-like patterning over 1-100 nm scales, or up to hundreds of lattice spacings. These sign-varying inhomogeneities or 'texturings' fundamentally affect local electronic, magnetic and structural properties, and appear to be intrinsic: arising from the coupling between degrees-of-freedom, rather than induced by extrinsic disorder.

Ferroelastic martensitic alloys like $\mathrm{FePd}$, and the more complex cuprates and manganites, typically have displacive first-order structural transitions [1, described by symmetryadapted strain tensor components as order-parameters (OP), with 1-10 nm criss-cross 'tweed' strain variations above, and 10-100 nm 'twins' below, the structural transition [1]. There is evidence for lattice/charge/spin coupling in both cuprates and manganites in: isotope effects [5]; doping-dependence of transition temperatures 11; polaron signatures with 4] temperature- and field-dependent four-lobe signatures in diffuse X-ray and neutron scattering;

(c) EDP Sciences 
EUROPHYSICS LETTERS

field-dependent wandering ribbons of high conductivity [1, 2, 6] ; and (fluctuating or static) stripe bubbles of modulated buckling-angle and spin 3. A strain-magnetization coupling is indicated by cross-responses [7: e.g., manganites that show colossal magneto-resistance (CMR) in magnetic fields also show colossal 'stresso-resistance' (CSR) under hydrostatic pressure.

The central questions are conceptual: what excitations result, when doped local charges in a (para- or antiferro-) magnetic background couple to a nonlinear elastic lattice that supports a structural transition? What is the origin of multiscale intrinsic inhomogeneities?

We adopt a Ginzburg-Landau (GL) approach, with three generic model assumptions: (1) The free energy is harmonic in the non-order-parameter strain components and nonlinear in the order-parameter strain (with multiple wells just above, and below, structural transition temperatures); (2) The charge density and magnetization variables couple locally, in symmetry-allowed ways, to different symmetry-adapted strain-tensor components, (and can then act like local internal stresses or temperatures); (3) The charge carriers deforming a unitcell do not damage the lattice by generating defects such as dislocations or vacancies (so there is a smoothly compatible matching to the farther, and decreasingly-strained, unit-cells). Longrange isotropic Coulomb potentials, with or without, or with electron-phonon coupling, have been invoked to explain nanoscale charged stripe patterns [8]. However, our central point here is that charges and spins locally coupled to strains of a nonlinear lattice, can induce multiscale mutual texturings through anisotropic long-range strain-strain forces (themselves Coulombic at a chemical-bond level [9]), that arise from generic elastic compatibility constraints [10].

GL model: 1. Strain Free Energy: We consider a two-dimensional (2D) first-order squareto-rectangular transformation as a surrogate for 3D tetragonal-orthorhombic [1] structural transitions; extensions to $3 \mathrm{D}$ are possible [11. The Cauchy strain tensor $\underline{E}$ linear in the displacement $\vec{u}$, is $E_{\mu \nu}=(1 / 2)\left(\Delta_{\mu} u_{\nu}+\Delta_{\nu} u_{\mu}\right)$, where $\Delta_{\mu}$ is a discrete derivative in the $\mu=x, y$ directions, and small 'geometric' nonlinearities are ignored. The symmetry-adapted strains are the 'rectangular' or deviatoric $\varepsilon \equiv(1 / \sqrt{2})\left(E_{x x}-E_{y y}\right)$, compressional $e_{1}=(1 / \sqrt{2})\left(E_{x x}+E_{y y}\right)$, and shear $e_{2}=E_{x y}$, strains, respectively. The invariant free energy is

$$
F=F^{(1)}\left(\varepsilon, e_{1}, e_{2}\right)+F^{(2)}(m)+F^{(\text {coupling })}\left(n, m, \varepsilon, e_{1}\right),
$$

with all quantities scaled [1] to be dimensionless. The strain contribution is

$F^{(1)}=\left(a_{0} / 2\right) \sum_{\vec{r}}(\vec{\Delta} \varepsilon)^{2}+F_{0}(\varepsilon)+F_{c s}\left(e_{1}, e_{2}\right)$, where $\sqrt{a_{0}}$ is a strain variation length. The Landau term is sixth-order in the deviatoric OP strain $F_{0}=(\tau-1) \varepsilon^{2}+\varepsilon^{2}\left(\varepsilon^{2}-1\right)^{2}$. Here $\tau(T)=\left(T-T_{s c}\right) /\left(T_{s}-T_{s c}\right)$ is a scaled temperature, and for $4 / 3>\tau>0, F_{0}$ has triple wells that become degenerate at $\tau\left(T=T_{s}\right)=1$, reducing to double wells for $T<T_{s c}$ or $(\tau<0)$. The compression/shear $(c s)$ terms are harmonic, $F_{c s}=\sum_{\vec{r}} \frac{1}{2} A_{1} e_{1}^{2}(\vec{r})+\frac{1}{2} A_{2} e_{2}^{2}(\vec{r})$.

2. Charge and Magnetization Free Energy: The free energy for a magnetization variable $m(\vec{r})$ is $F^{(2)}(m)=\sum_{\vec{r}} f\left[\left(\frac{a_{m}}{2}\right)(\vec{\Delta} m(\vec{r}))^{2}+\left(T-T_{c m}\right) m^{2}(\vec{r})+\frac{1}{2} m^{4}(\vec{r})\right]-h m$. Here $f$ is a magnetic/elastic energy ratio, $h\left(\sqrt{a_{m}}\right)$ is a magnetic field (length), and the zero-doping magnetic transition temperature $T_{c m}<T_{s}$. The symmetry-allowed couplings are

$$
F^{(\text {coupling })}\left(n, m, \varepsilon, e_{1}\right)=\sum_{r} A_{n \varepsilon} n \varepsilon^{2}+A_{n m} n m^{2}+\left[A_{n 1} n e_{1}+A_{m 1} m^{2} e_{1}+p_{1}(\vec{r}) e_{1}\right],
$$

where $p_{1}(\vec{r})>0$ is an external compressional stress. The $A_{n \varepsilon} n \varepsilon^{2}$ term is like a local temperature $\sim \tau \varepsilon^{2}$, and for $A_{n \varepsilon}>0$, the charge favours the high-temperature $\varepsilon=0$ unit-cell symmetry. The $A_{m 1} m^{2} e_{1}$ term acts like a local stress $\left(\sim m^{2}\right)$ for $e_{1}$, and like a local temperature $\left(\sim e_{1}\right)$ for $m^{2}$. We choose $A_{m 1}>0$, favouring $m^{2}$ when spins are closer $\left(e_{1}<0\right)$. The $m^{2}$ coefficient in $F$ defines an effective local temperature deviation $\tau_{c m}(T, \vec{r}) \equiv f\left[T-T_{c m}^{e f f}(\vec{r})\right] / A_{m 1} \equiv$ $e_{1}+\left[f\left(T-T_{c m}\right)+A_{n m} n\right] / A_{m 1} \equiv \delta e_{1}(T, \vec{r})$. This implies a temperature-dependent percolation: 
on warming, the compressed $m \neq 0$ regions below transition $\left(\tau_{c m}(T, \vec{r})=\delta e_{1}(T, \vec{r})<0\right)$ will shrink. We now mimic manganites and cuprates by further model choices of parameter signs and interpretations of variables.

(i) For 'manganites', with a zero-doping nonferromagnetic $T_{c m}=0$ parent compound, we focus for simplicity on the magnetization-inducing mobile electrons produced by doping, of concentration $x_{e}=\left\langle n>\right.$ where the number density $n(\vec{r})=\sum_{i}\left(\kappa^{2} / 2 \pi\right) e^{-\kappa\left|\vec{r}-\vec{r}_{i}\right|}$ is a sum over normalized single-charge profiles at sites $i$, with $2 / \kappa$ the intersite tunneling length. Here the $A_{n m} n m^{2}$ term with $A_{n m}<0$ means that mobile electrons lock onto and align the ferromagnetic $m(\vec{r})$ core spins (mimicking a double exchange/ Hund's rule, term). The mobile electrons can induce an effective local [6] Curie temperature, $T_{c m}^{e f f}(\vec{r})=\left(-A_{n m} n-A_{m 1} e_{1}\right) / f>$ 0 . Since metallicity shrinks unit-cell volume, we take the $A_{n 1} n e_{1}$ term with $A_{n 1}>0: n(\vec{r})$ favours compression, $e_{1}<0$.

(ii) For 'cuprates', $n(\vec{r})$ is the local hole number density of doping fraction $x_{h}=<n>$ into an antiferromagnetic parent compound with Néel temperature $T_{c m} \neq 0$. With $A_{n m}>0$ in $A_{n m} n m^{2}$, the staggered magnetization $m(\vec{r})$ is reduced by hole doping. As the structural transition temperature also decreases rapidly with doping [1], we take $A_{n \varepsilon}>>1$. Since holes repel lattice ions, the $A_{n 1} n e_{1}$ term has $A_{n 1}<0: n(\vec{r})$ favours expansion, $e_{1}>0$.

3. Elastic Compatibility and Anisotropic Long-range Potentials: The St. Venant's compatibility condition [10] expresses the no-defect constraint as $\vec{\Delta} \times(\vec{\Delta} \times \underline{\underline{E}})^{\dagger}=0$. (This is analogous to a $\vec{\Delta} \cdot \vec{B}=0$ no-monopole condition in electromagnetism.) In $2 \mathrm{D}$ we have $12 \vec{\Delta}^{2} e_{1}-\sqrt{8} \Delta_{x} \Delta_{y} e_{2}=\left(\Delta_{x}^{2}-\Delta_{y}^{2}\right) \varepsilon$, linking OP and non-OP strains. Minimizing the free energy $F_{c s}$ with respect to $e_{1,2}$ while maintaining the compatibility constraint, we find $e_{1,2}(\vec{k})=B_{1,2}(\vec{k}) \varepsilon(\vec{k})$, where $B_{1}(\vec{k})=\left[k_{x}{ }^{2}-k_{y}{ }^{2}\right] k^{2} /\left[k^{4}+\left(8 A_{1} / A_{2}\right)\left(k_{x} k_{y}\right)^{2}\right]$ and $B_{2}(\vec{k})=$ $-\left(A_{1} / A_{2}\right)\left[\sqrt{8} k_{x} k_{y} / k^{2}\right] B_{1}(\vec{k})$. Substituting back, the seemingly innocuous harmonic $F_{c s}$ yields 11,12 the crucial ALR potential, encoding unit-cell fourfold symmetries,

$$
F_{c s}=\left(A_{1} / 2\right) \sum_{\vec{k}} U(\hat{k})|\varepsilon(\vec{k})|^{2} ; \quad U(\hat{k})=\left[\left(k_{x}{ }^{2}-k_{y}{ }^{2}\right)^{2} /\left[k^{4}+\left(8 A_{1} / A_{2}\right)\left(k_{x} k_{y}\right)^{2}\right]\right.
$$

while (2) yields nonlocal couplings to the OP. Here $U(\hat{k})$ depends on the direction $\hat{k}$ and clearly favors $\hat{k_{x}}=\hat{k_{y}}$ diagonal strain textures, with a Meissner-like $e_{1,2}=0$ expulsion [1]. In coordinate space, with $\hat{r} \cdot \hat{r}^{\prime}=\cos \left(\theta-\theta^{\prime}\right)$, the potential $U\left(\vec{r}-\vec{r}^{\prime}\right) \sim \cos 4\left(\theta-\theta^{\prime}\right) /\left|\vec{r}-\vec{r}^{\prime}\right|^{D}$ has sign-variation supporting elastic frustration, with the OP strain at a point receiving conflicting ("ferro/antiferro") instructions from other surrounding strains. The power-law $(D=2)$ decay arises from $U(\hat{k})$ being scale-free $(|\boldsymbol{k}|$-independent) at long wavelengths, rather than from proximity to some critical point.

Our central physical idea is quite simple. Suppose, among (symmetry-broken) rectangular unit cells $\varepsilon(\vec{r})=1$, that a single unit-cell is made square, $\varepsilon(\vec{r})=0$. To maintain lattice integrity, the neighboring (and further) unit cells must also deform, with an admixture of nonOP strains. As shown in simulations of a nonlinear-strain model under local external stress, for $A_{1,2}>>1$ the large non-OP energy costs can make it profitable to locally summon up the (degenerate) competing structure, in energy-lowering higher elastic multipoles: a process of adaptive elastic screening 11. Thus a charge, acting as a local internal stress, can produce an unusual sign-varying (i.e. textured) extended polaron modulated by the anisotropic $U\left(\vec{r}-\overrightarrow{r^{\prime}}\right)$, with coupled fields like $m(\vec{r})$ also sign-varying. This polaronic elasto-magnetic texture, or 'pemton' arises from compatibility and competing ground states, and differs from the more familiar magneto-elastic polaron [13] that deforms a single lattice structure.

We now choose parameters. For the martensite $F e P d$, physical values 12 can be scaled 
[11] to be dimensionless, and $\varepsilon=e_{1}=e_{2}=1$ correspond to strains $\sim 0.02$; the scaled stress $p_{1}=1$ (magnetic field $h=1$ ) corresponds to $\sim 0.02 \mathrm{GPa}(\sim 0.25$ Tesla); and the non-OP and OP elastic constants are $A_{1}=150, A_{2}=300$. We take as illustrative, $A_{1}=$ $50, A_{2}=105, \sqrt{a_{0}}=0.5, \sqrt{a_{m}}=1, \kappa=2, T_{s}=1, T_{s c}=0.8, f=0.3, A_{m 1}=+5$, and specific 'manganite' ('cuprate') model parameter sets as $T_{c m}=0, A_{n m}=-1, A_{n 1}=+5, A_{n \varepsilon}=+2$ $\left(T_{c m}=0.6, A_{n m}=+9, A_{n 1}=-5, A_{n \varepsilon}=20\right)$. This is a regime of globally weak magnetism, relatively strong electron-phonon coupling, and dominant compatibility forces.

Simulation of Textures: The free energy $F=F(n, m, \varepsilon)$ minimum is found by the overdamped limit of a general ferroelastic dynamics [11]:

$$
\dot{\varepsilon}=-\frac{\partial F}{\partial \varepsilon} ; \quad \dot{m}=-\frac{\partial F}{\partial m} .
$$

We show selected relaxed profiles [14] of $\varepsilon, e_{1}, m$, with both $\vec{k}$ and $\vec{r}$ plots needed for a full understanding. Figure 1 shows strain plots in coordinate space (of $e_{1}(\vec{r})$ ) and in Fourier space (of $|\epsilon(\vec{k})|^{2}$ ) due to a single charge for 'manganite' parameters. The ne local stress term would by itself produce a bare single-sign strain, so adaptive elastic screening is responsible for the observed textured polaron or 'pemton'. The butterfly-like quadrupolar (and essentially cancelling) lobes of both signs in strain and magnetization, extends over $\sim 20$ lattice spacings, explicitly illustrating the concept [1, 3, 6] of magnetic and structural [2] 'nano-scale phase separation'. Bi-pemtons from nearby charges form stripe-like segments.

For increasing random doping at $T=0.5$, the average magnetization $<m>$ rises sharply from zero for $x_{e}>0.13$, to e.g., $\left\langle m>=-0.21\right.$ at $x_{e}=0.15$, with symmetry-breaking in the $m(\vec{r})$ carried by the pemtons. Fig. 2 shows the mutually deforming multi-pemtons, forming meandering ribbons of expanded/compressed unit-cell strain $e_{1}(\vec{r})$ or oriented spins $m(\vec{r})$. Fourier space plots, e.g. of $|\varepsilon(\vec{k})|^{2}$ (or $\left|e_{1}(\vec{k})\right|^{2}$ ), show a four-lobe shape (as in the single-pemton case) reminiscent of diffuse X-ray and neutron scattering [4]. A signature of compatibility forces is the $\vec{k} \rightarrow-\vec{k}$ inversion symmetry (in the squared strain) relating most of the even finescale crinkles. Fig. 2 also shows a reduction, on warming, of both $\langle m\rangle$ and of $m(\vec{r})$ percolation [2] 15], at fixed doping.

Figure 3 shows the effects on the composite multipemton of an external spatially varying compressional stress $p_{1}(\vec{r})$ or magnetic field $h(\vec{r})$, with four quadrants in each picture from the long wavelength modulation. The diagonal plots show the $p_{1} \rightarrow e_{1}, h \rightarrow m$ or direct responses. The off-diagonal plots show the $p_{1} \rightarrow m, h \rightarrow e_{1}$ or cross responses, e.g. fine cloud-like gradations reminiscent of STM/TEM pictures [2]; and enhanced magnetic percolation under stress [7. The CMR/CSR 'colossal' effects can be understood through the "compressed magnetic $\sim$ metallic" interconnections, with fields/stresses locally tipping the delicate balance between opposite-sign large values (see vertical scales) of $m(\vec{r}), e_{1}(\vec{r})$, thus opening up conducting channels both in magnetization (via double exchange), or in compression (via enhanced tunneling). The rich phase diagram [1] of charge- (or pemton-) ordered states could arise from the orienting long-range compatibility forces [16]. Charge profile relaxation into locally compressed regions would describe orbital ordering, or coexisting localized/extended electronic states.

For 'cuprate' parameters, the Néel temperature is $T_{c m}=0.6$, and at $T=0.5$, the parent compound has a uniform $m$. Figure 4 shows that a 'cuprate' single pemton is smaller, stronger, and sharper than Fig.1. The 'cuprate' multi-pemton with $x_{h}=0.1$ aligns $\varepsilon(\vec{r})$ into diagonal parallel ribbons, forming bubbles of stripes [3], with changes for higher doping.

In summary, the central insight from our model is that under doping perturbation , a nonlinear lattice can produce 'intrinsic inhomogeneities', that are not quenched-defect random 
spots, but rather, self-organized annealed-texture responses, induced by the multiscale effects of local lattice-integrity constraints. These composite textures vary with $T, p_{1}$ or $h$.

The compatible, inter-cell large-strain texturings must be supported by intra-cell deformations of the atomic bases ('microstrain'), reflected in bond angle/length distributions [3 9], and so will be relevant for complex electronic oxides, with atomic bases of tiltable perovskite octahedra (that have directionally bonded transition-metal ions, and polarizable/deformable oxygens 1]). Further electronic structure studies would allow for both electronic and ionic optimizations. Further theoretical work includes exploring parameter space extensively, in 2D and $3 \mathrm{D}$, in overdamped or other 11 regimes; adding charge-hopping dynamics and charge-profile relaxations; and strain-related microscopic modelling e.g. of plane bucklings [17 induced by octahedral tilts. Further experimental work should include STM mapping of symmetryadapted strains to seek pemton signatures.

It is a pleasure to thank Seamus Davis, Carlos Frontera, and Venkat Pai for useful conversations. This work was supported by the U.S. Department of Energy.
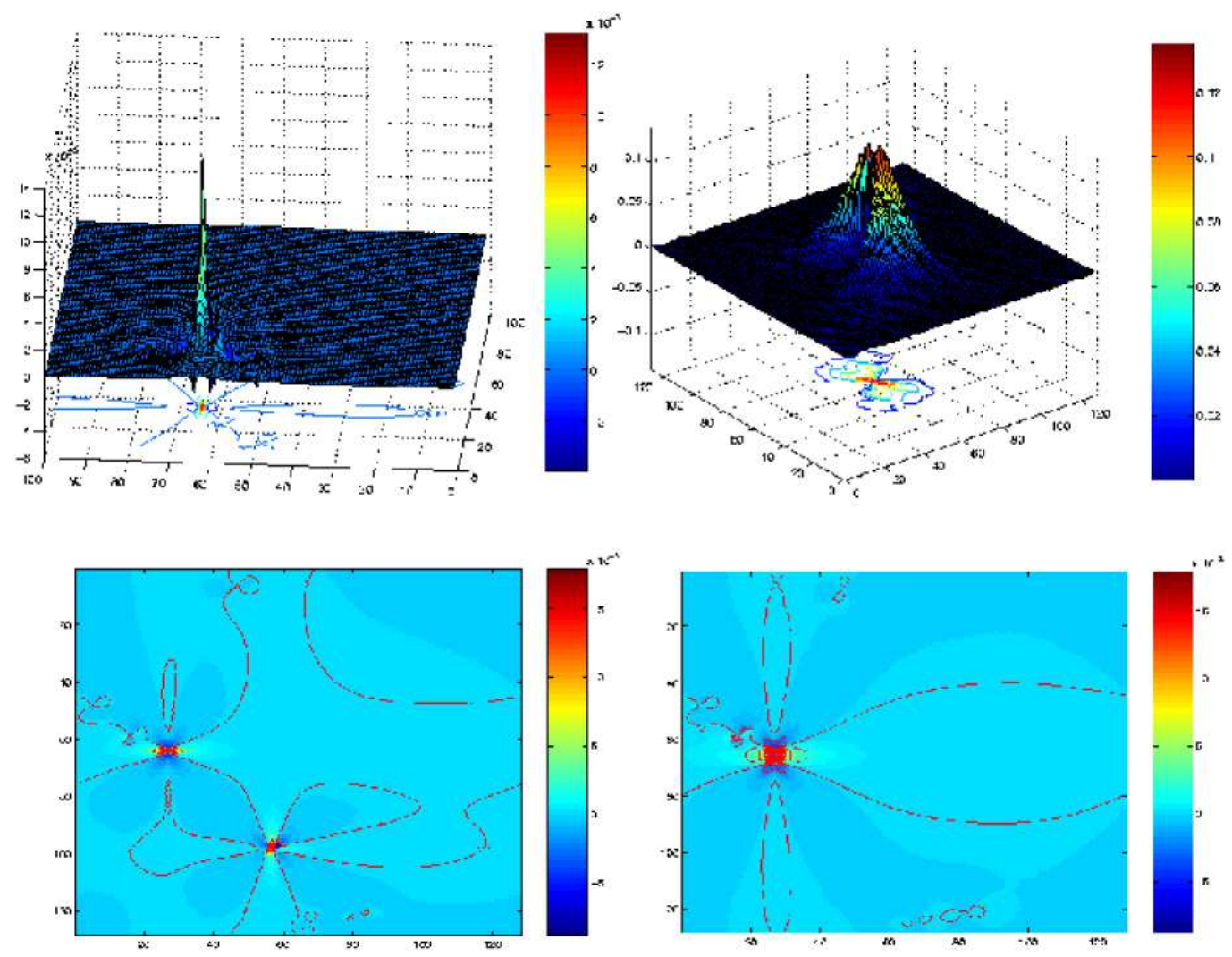

Fig. 1 - Single polaronic elasto-magnetic texture or pemton state for 'manganite' parameters: Temperature $T=0.5$. All rows are read left to right. Top row: relief and contour plots of the compressional $e_{1}(\vec{r})$ strain $\times 10^{-3}$; and Fourier space deviatoric strain of $|\varepsilon(\vec{k})|^{2}$. Bottom row: colour plot of $e_{1}(\vec{r})$ for separated and nearby pemtons. Note that bi-pemtons form stripe segments. 

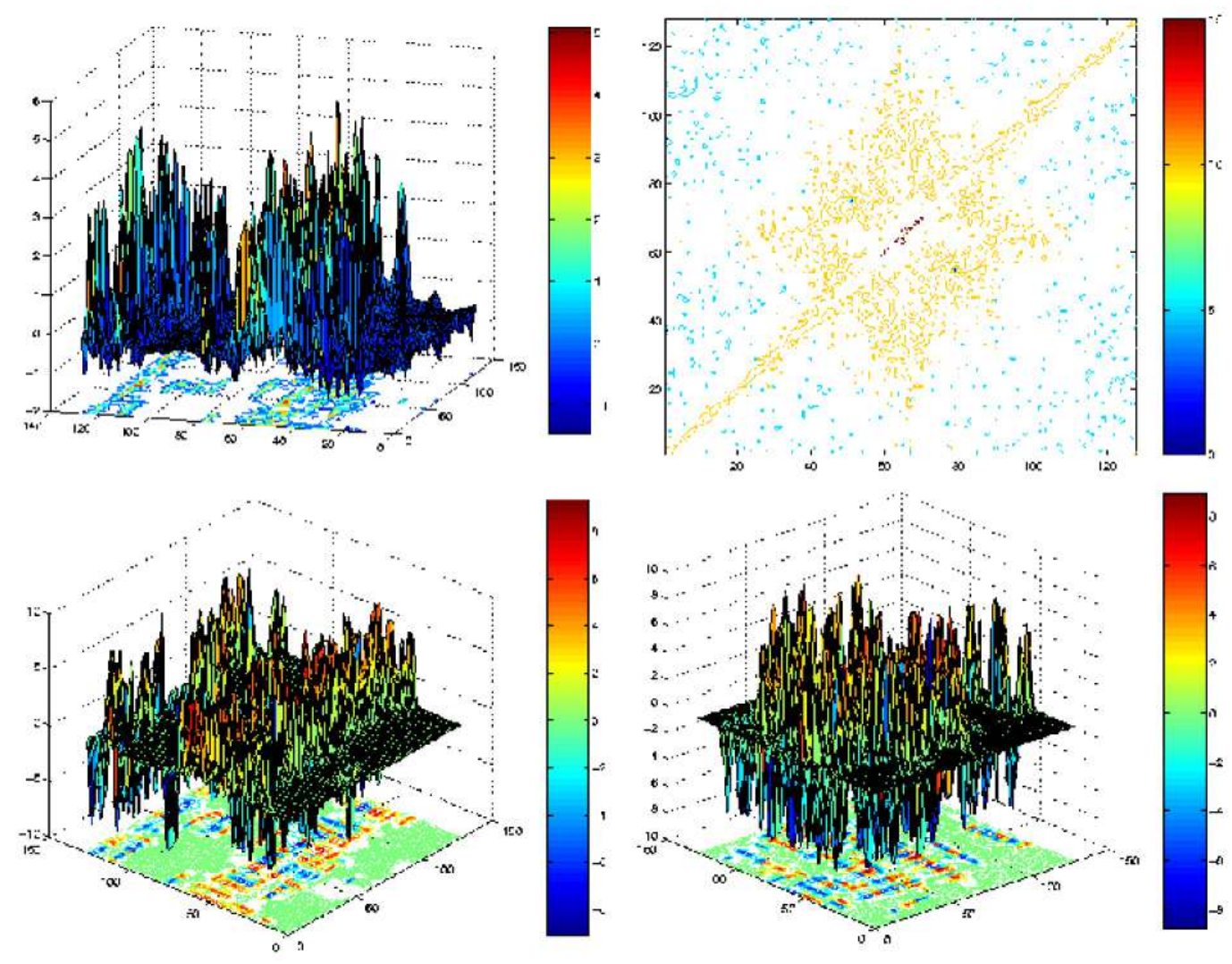

Fig. 2 - Multipemton states for 'manganite' parameters: Electron doping fraction $x_{e}=0.15$. Relief/contour plots; all rows read left to right. Top row: $T=0.5$. Plot of the compressional strain $e_{1}(\vec{r})$. Plot in Fourier space of multiscale $|\varepsilon(\vec{k})|^{2}$ (log scale). Bottom row: magnetization $m(\vec{r})$ for $T=0.5$ and 0.8 , with large values compensating to yield $\langle m\rangle=-0.21$ and -0.06 , respectively.

\section{REFERENCES}

[1] Stripes 2000, eds. N.L. Saini and A. Bianconi, Int. J. Mod. Phys., 14, 3289 (2000); Colossal Magnetoresistance and Related Properties, eds. B. Raveau and C.N.R. Rao, (World Scientific, Singapore 1998); E.K.H. Salje Phase Transitions in Ferroelastic and Coelastic Solids, (Cambridge University Press, Cambridge, UK 1990); N.F. Mott in Polarons and Bipolarons in High$T_{c}$ Superconductors, ed. E.K.H. Salje, A.S. Alexandrov, and W.Y. Liang, (Cambridge University Press, Cambridge, UK, 1995); A.J. Millis, Nature, 399, 147 (1998); Nanoscale Phase Separation and Colossal Magnetoresistance, ed. E. Dagotto, (Springer, 2003); Lattice Effects in High- $T_{c}$ Superconductors, eds. Y. Bar-yam, T. Egami, J. Mustre-de Leon, and A.R. Bishop, (World Scientific, 1992); Intrinsic Multiscale Structure and Dynamics in Complex Electronic Oxides, eds. A.R. Bishop, S.R. Shenoy and S. Sridhar, (World Scientific, 2003).

[2] M. Faeth, S. Friesen, A.A. Menovsky,Y. Tomioka, J. Aarts,J.A. Mydosh, Science 285, 1540 (1999); M. Uehara, S. Mori, C.H. Chen, and S-W. Cheong, Nature 399, 560 (1999);M. Uehara and S-W. Cheong, Europhys. Lett. 52, 674 (2000).

[3] A. Bianconi, N.L.Saini, A.Lanzara, M.Missori, T.Rosetti, H.Oyanagi, H.Yamaguchi, K.Oka, and T.Ito, Phys. Rev. Lett. 76, 3412 (1996); E.S. Bozin, G.H. Kwei, H. Takagi, and S.J.L. Billinge, Phys. Rev. Lett. 84, 5856 (2000). 

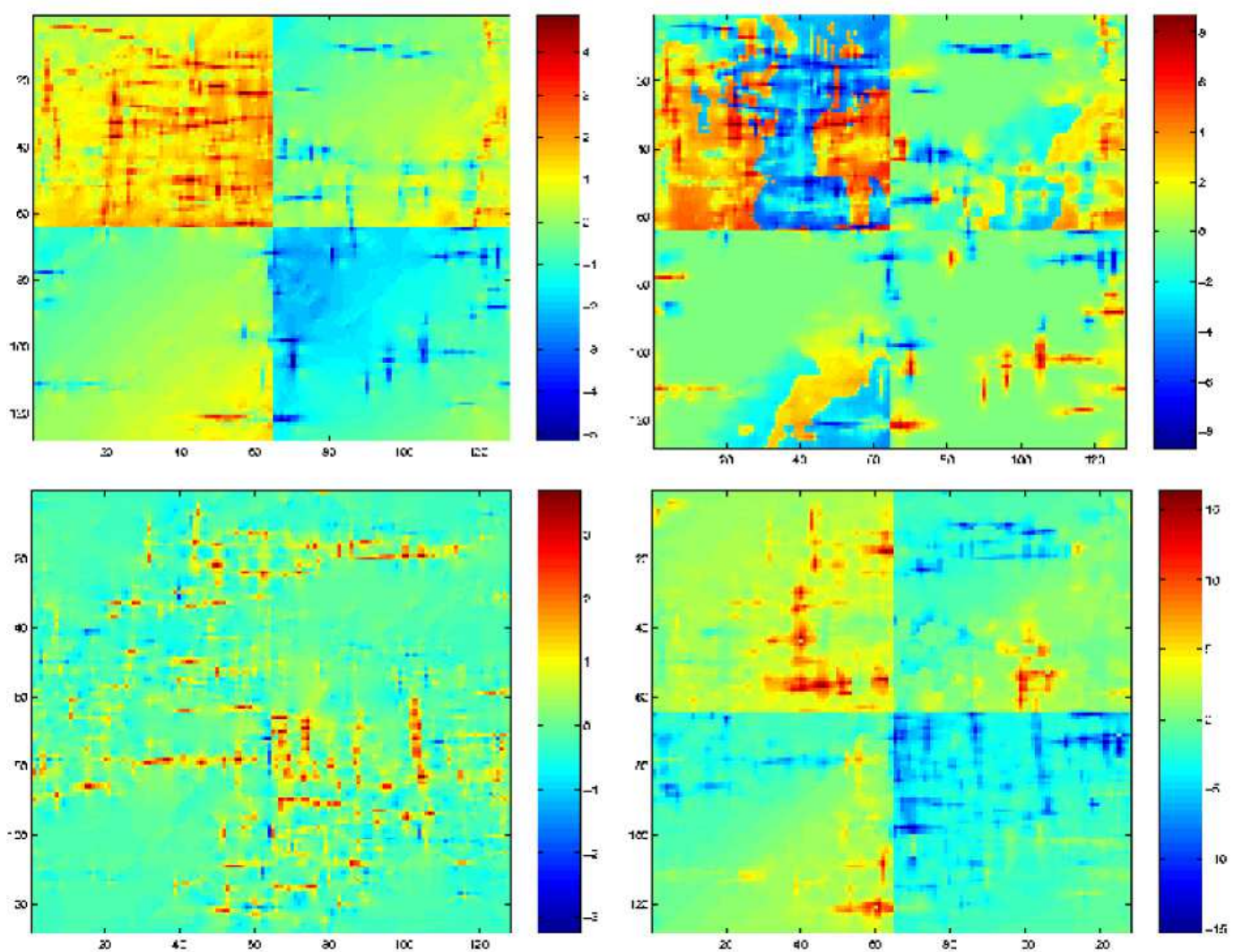

Fig. 3 - Response of 'manganite' multi-pemton states to external stress and magnetic field: Doping $x_{e}=0.15$, and $T=0.5$, as in the top left and bottom left of Fig. 2. Colour plots, with all rows read left to right, with four quadrants in each, from the longwavelength variation of stress $p_{1}(\vec{r})=p_{1} f(\vec{r})$ and magnetic field $h(\vec{r})=h f(\vec{r})$, where $f(\vec{r})=\frac{1}{2}[\cos (2 \pi x / N)+\cos (2 \pi y / N)]$. Top row: $e_{1}(\vec{r})$ and $m(\vec{r})$ under stress, with $p_{1}=20(\sim 0.4 \mathrm{GPa})$. Bottom row: $e_{1}(\vec{r})$ and $m(\vec{r})$ in a magnetic field, with $h=10(\sim 2.5$ tesla $)$. Note that $p_{1}(\vec{r})$ and $h(\vec{r})$ change both $e_{1}$ and $m$, at all scales.

[4] L. Vasiliu-Doloc, S. Rosenkranz, R. Osborn, S.K. Sinha, J.W. Lynn, J. Mesot, O.H. Seeck, G. Preosti, A.J. Fedro, and J.F. Mitchell, Phys. Rev. Lett. 83, 4393 (1999); S. Shimomura, N. Wakabayashi, H. Kuwahara and Y. Tokura, Phys. Rev. Lett., 83, 4389 (1999).

[5] G-M. Zhao, K. Conder, H. Keller and K.A. Mueller, Nature 381, 676 (1996); G.-M. Zhao, K.K. Singh, and D.E. Morris, Phys. Rev. B 50, 4112 (1994); D. Zech, H. Keller, K.A. Mueller, K. Conder, E. Kaldis, E. Liarokapis, and N.Poulakis, Nature 371, 681 (1994); D. Rubio Temprano, J. Mesot, S. Janssen, K. Conder, A. Furrer, H. Mutka, and K.A. Mueller, Phys. Rev. Lett. 84, $1990(2000)$.

[6] J. Burgy, M. Mayr, V. Martin-Mayor, A. Moreo and E. Dagotto, Phys. Rev. Lett. 87, 277202 (2001); M.B. Salamon, P. Lin, and S.H. Chun, Phys. Rev. Lett. 88, 197203 (2002).

[7] Y. Hwang, T.M.Palstra , S-W. Cheong, and B. Batlogg, Phys.Rev.B 52, 15046 (1995); A.N. Lavrov, S. Komiya, and Y. Ando, Nature 418, 385 (2002). S.B. Ogale, V.Talyansky, C.H.Chen, R.Ramesh, R.L.Greene, and T. Venkatesan, Phys. Rev. Lett. 77, 1159 (1996).

[8] J. Zaanen and O. Gunnarson, Phys. Rev. B 40, 7391 (1989); S.A. Kivelson and V.J. Emery, Physica C 235, 189 (1999); F. Kusmartsev, Phys. Rev. Lett. 84, 5026 (2000).

[9] C. Zener, Phys.Rev.71, 846 (1947); J. McAllister, and J.P. Attfield, Phys.Rev.B66, 04514 (2002) 

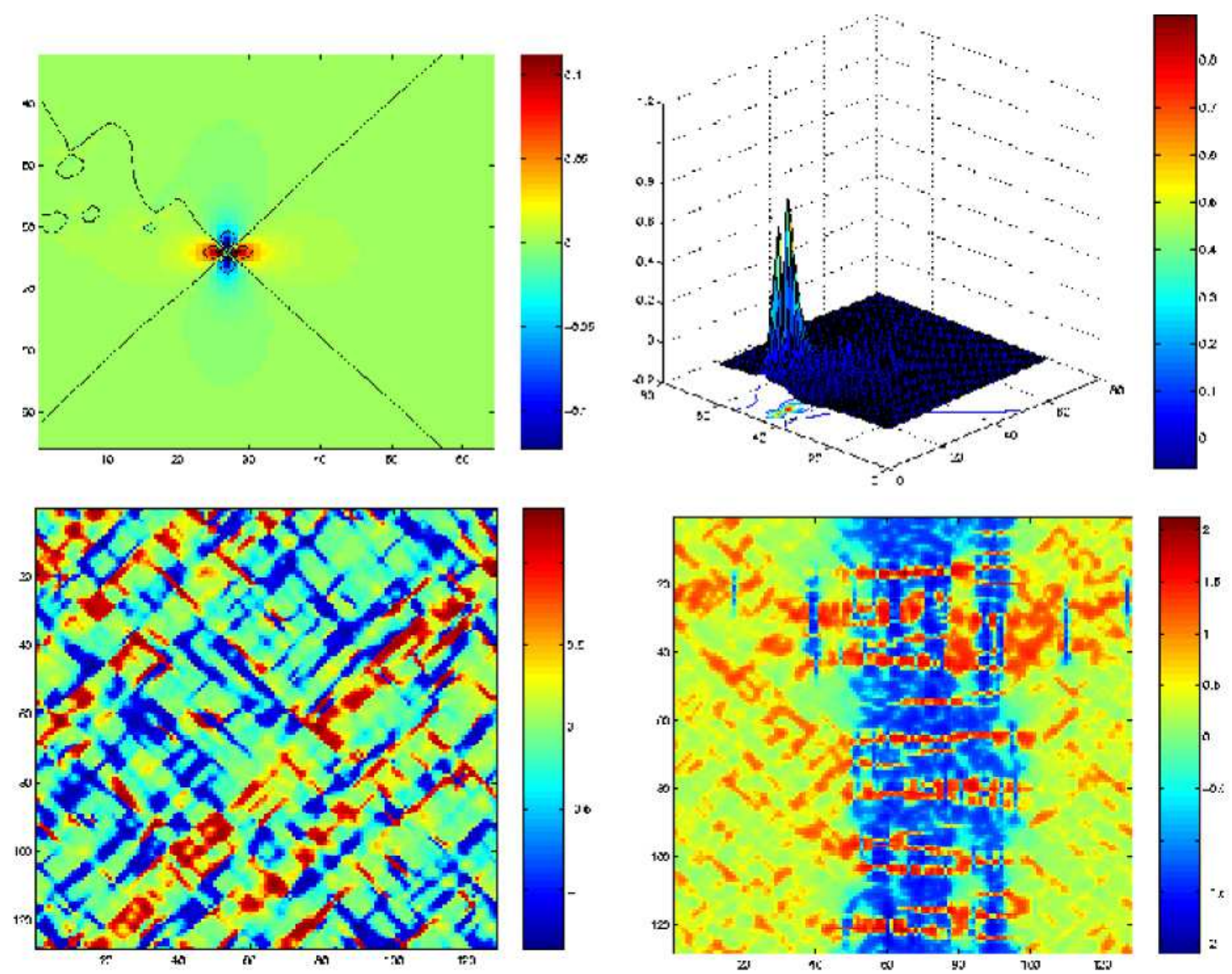

Fig. 4 - Pemtons for 'cuprate' parameters: Temperature $T=0.5$; parent compound (subtracted) has Néel temperature $T_{c m}=0.6$. All rows read left to right. Top row: Colour plot of single-pemton $e_{1}(\vec{r})$. Relief/contour plot of staggered magnetization $m(\vec{r})$ with $\langle m\rangle=3 \times 10^{-3}$. Bottom row: Colour plots of $\varepsilon(\vec{r})$ for random pemtons with hole fractions $x_{h}=0.1$ and 0.2 .

; K. Iwano, Phys. Rev. B 64, 184303 (2001).

[10] M. Baus and R. Lovett, Phys. Rev. Lett. 65, 1781 (1990); Phys. Rev. A 44, 1211 (1991); D.S. Chandrasekharaiah and L. Debnath, p. 218, Continuum Mechanics, (Academic, San Diego, 1994).

[11] S.R.Shenoy, T.Lookman, A.Saxena, and A.R.Bishop, Phys. Rev. B 60, R12537 (1999); K.Ø. Rasmussen, T.Lookman, A.Saxena, A.R.Bishop, R.C.Albers, and S.R.Shenoy, Phys. Rev. Lett. 87, 055704 (2001) , T. Lookman, S.R. Shenoy, K. Rasmussen, A.Saxena and A.R. Bishop, Phys. Rev. B 67, 021301 (2003).

[12] S.Kartha, J.A. Krumhansl, J.P. Sethna and L. Wickham, Phys. Rev. B 52, 803 (1995).

[13] H. Roeder, J. Zhang, and A.R. Bishop, Phys. Rev. Lett. 76, 1356 (1996).

[14] We consider $128 \times 128$ systems and periodic boundary conditions, with charge profiles introduced through their site Fourier transforms. We first relax the strain by itself, from initial $\varepsilon(\vec{r}, t=0)$ random within \pm 0.2 . The magnetization is then coupled and relaxed from $m(\vec{r}, t=0)$ fluctuations around the zero-doping meanfield value. This 'parent compound' has (unequal-separation) diagonal domain walls, usually with a few metastable kinks. Charges are added at fixed random positions (but forbidding double occupancy). Further relaxations are up to $3 \times 10^{4}$ time steps, 
with step size $\Delta t \sim 0.01$, using as equilibration diagnostics, the free energy and its 'force' derivatives, and variable averages and $\mathrm{max} / \mathrm{min}$ values. The parent background is then subtracted. Signatures of (charge-deformed) background kinks and twins can show up in the Figs.

[15] On cooling, nonzero (positive and negative) $m(\vec{r})$ 'metallic' regions could possibly percolate across the system at the same insulator-metal transition temperature. Then symmetry-breaking to a nonzero $<m>$ between these competing-sign backbones could occur at a lower ferromagnetic temperature. Alternatively, for other parameters, isolated islands could order $(<m>\neq 0)$ before percolation, inverting the sequence of the transitions.

[16] In our model, for periodic charge arrays, momentum conservation on the $n, m, e_{1}$ couplings of (3) imply that dominant (single) wavevectors are related by $k_{n}=2 k_{m}=k_{1}$.

[17] S.R.Shenoy, V.Subrahmanyam, and A.R.Bishop, Phys. Rev. Lett., 79, 4657 (1997); D. Mihailovic, V. Kabanov, and K.A. Müller, Europhys. Lett. 57, 254 (2002). 